\title{
Tissue Clearing and Deep Imaging of the Kidney using Confocal and Two-Photon Microscopy
}

\author{
Daniyal J Jafree* 1,2, David A Long 1, Peter J Scambler 1, Dale Moulding 2,3 \\ ${ }^{1}$ Developmental Biology and Cancer Programme, UCL Great Ormond Street Institute of \\ Child Health, University College London, London, UK \\ ${ }^{2} \mathrm{MB} \mathrm{PhD}$ Programme, Faculty of Medical Sciences, University College London, London, \\ UK \\ ${ }^{3}$ Light Microscopy Core Facility, UCL Great Ormond Street Institute of Child Health, \\ University College London, London, UK

\section{* Corresponding author:} \\ Daniyal J Jafree, \\ Room 222, \\ Developmental Biology and Cancer Programme, \\ UCL Great Ormond Street Institute of Child Health, \\ University College London, \\ London, WC1N 1EH, UK \\ Telephone: $+44(0) 2079052196$ \\ Email: daniyal.jafree.13@ucl.ac.uk
}

Running head: Confocal and two-photon deep renal imaging 


\begin{abstract}
Microscopic and macroscopic evaluation of biological tissues in three-dimensions is becoming increasingly popular. This trend is coincident with the emergence of numerous tissue clearing strategies, and advancements in confocal and two-photon microscopy, enabling the study of intact organs and systems down to cellular and sub-cellular resolution. In this chapter, we describe a wholemount immunofluorescence technique for labelling structures in renal tissue. This technique combined with solvent-based tissue clearing and confocal imaging with or without two-photon excitation, provides greater structural information than traditional sectioning and staining alone. Given the addition of paraffin embedding to our method, this hybrid protocol offers a powerful approach to combine confocal or two-photon findings with histological and further immunofluorescent analysis within the same tissue.
\end{abstract}

Key Words: Confocal, Imaging, Kidney, Three-Dimensional, Tissue Clearing, Two-Photon 


\section{INTRODUCTION}

With the advent of novel wholemount immunofluorescence strategies, and improvements in microscope objectives and their working distances, three-dimensional imaging and analysis is becoming increasingly popular. Previously, obtaining three-dimensional images of organs would require sectioning, imaging and alignment of serial sections to manually generate a zstack, which is time-consuming, laborious and technically challenging. Now, intact imaging of entire organs or small organisms has been made possible by the wealth of tissue clearing strategies that are now readily available, and the advancements in confocal and lightsheet imaging, enabling interrogation of intact biological structures down to cellular and even subcellular resolution. The principle of tissue clearing, also known as refractive index (RI) matching, and fluorescence imaging has been recently reviewed extensively [1,2], even in the context of the kidney [3]. As light enters tissue, it scatters due to interaction with electrons and subsequent release of spherical waves, with a concomitant slowing of the propagation of light through the tissue. The latter is the basis for the RI of the tissue. The aim of clearing tissues is to achieve minimal lateral scattering and enable all wavelengths of light to pass through the tissue [1]. These principles of RI matching have been integrated into pre-existing wholemount immunofluorescence protocols, and most of these new protocols can be broken down into four steps [4]. Firstly, tissue may need to be pre-treated by, for example, decolourising, bleaching or other methods to quench autofluorescence. The second step enables penetration of subsequent reagents in the protocol and is critical for the immunolabelling and clearing of large volumes of tissue. This is achieved by permeabilising agents, including detergents such as Triton X-100, or solvents such as dimethyl sulfoxide (DMSO). At this stage, some protocols also make use of hydrogel embedding to preserve tissue morphology and minimise protein loss. Thirdly, tissues can be immunolabelled, either passively by diffusion, or aided by manipulation of pressure or electric fields within the reaction vessel. The final stage is clearing, after which volume imaging of the tissue is possible.

There are numerous tissue clearing techniques now available, each with their own advantages and disadvantages. Broadly, tissue clearing strategies can be grouped into four categories: solvent-based, simple immersion, hyperhydration and hydrogel embedding. Some protocols combine different approaches from each category. Importantly, many of these protocols have been conceived, adapted and optimised in neural tissue, which appears to be the most popular 
application for tissue clearing. Neural tissue has a high lipid content, therefore delipidation is a priority for successful transparency. We have tried several of these protocols for clearing renal tissue with unsatisfactory results, including ScaleA2 [5], ScaleS [6] and ClearT2 [7]. It is unclear whether this is due to different properties of renal tissue, differences in reagents, insufficient incubation times or subtle variations in experimental method. Regardless, the strategy that has yielded the most success, and one we have recently begun to implement regularly in our laboratory, is an older solvent-based approach, using a hybrid solution of benzyl alcohol and benzyl benzoate (termed BABB). Implemented in 2007 for the clearing and imaging of whole mouse brains, mouse embryos or fruit flies [8], BABB has largely gone out of fashion for neural tissue clearing due to its toxicity, incompatibility with water immersion objectives and quenching of fluorescent proteins. BABB, however, remains a quick and effective method for clearing renal tissue, having recently been used for applications such as counting podocytes in mouse glomeruli [9] or comparing the developmental features of mouse and human embryonic kidneys [10].

In this Chapter we describe a wholemount immunofluorescence labelling protocol that was designed and refined for solvent-based clearing using dibenzyl ether [11,12]. This protocol, the latest iteration of which is termed immunolabelling-enabled three-dimensional imaging of solvent-cleared organs (iDISCO+) [13], is a powerful and quick tool for the immunolabelling renal tissue. We have combined the iDISCO pipeline with BABB-based clearing, given its low cost, ability to rapidly clear renal tissue and high surface tension, making it useful when mounting samples between slides in preparation for confocal imaging. After acquisition of three-dimensional images, an image analysis pipeline can be developed using the range of commercial and open access image analysis software now available [13-16], or custom approaches can be applied for renal images, such as TreeSurveyor for quantifying ureteric bud branching or automated segmentation and counting of glomeruli $[17,18]$. Inspired by previous work using multidimensional approaches to study renal branching morphogenesis $[19,20]$, we have supplemented this protocol with a strategy to process and embed the immunolabelled and cleared renal tissue in paraffin. By combining this with traditional histology and immunohistochemistry techniques, one can assess three-dimensional structures of interest within the kidney using confocal imaging, whilst also obtaining information about renal histology for disease phenotyping and can supplement observations in confocal imaging with further immunolabelling of serial sections. Thus using our protocol, a wealth of data can be gathered from a single kidney (Figure 1). 


\section{MATERIALS}

Prepare all reagents in autoclaved glass containers or microcentrifuge tubes. All newly prepared reagents should be mixed thoroughly until fully dissolved. For reagents, individual storage time and safety considerations are provided in Table 1 (see Note 1).

\section{1 iDISCO+ wholemount immunofluorescence}

Many of the reagents here are 1 x phosphate-buffered saline (PBS)-based. We add $0.02 \%$ sodium azide to our stock $1 \mathrm{x}$ PBS solution prior to using it in subsequent reagents to prevent microbial growth. The protocol outlined below is based on those found on the iDISCO website (see Note 2 ) with some modifications.

\subsubsection{Reagents}

1. $1 \times$ PBS made in double distilled water (ddH20):

2. $4 \%(\mathrm{w} / \mathrm{v})$ paraformaldehyde (PFA) in $1 \mathrm{x}$ PBS: Dissolve $4 \%(\mathrm{w} / \mathrm{v})$ paraformaldehyde in $1 \mathrm{x}$ PBS in a fume hood under heat

3. Bleaching solution: Methanol with $5 \%(\mathrm{v} / \mathrm{v})$ of $30 \%$ hydrogen peroxide solution

4. PTx.2: 1 x PBS with $0.2 \%(\mathrm{v} / \mathrm{v})$ Triton X-100

5. PTwH: $1 \times$ PBS with $0.2 \%(v / v)$ Tween-20 and $0.1 \%(\mathrm{v} / \mathrm{v})$ heparin stock solution

6. Blocking solution: PTx.2 with $10 \%(\mathrm{v} / \mathrm{v})$ DMSO and $6 \%(\mathrm{v} / \mathrm{v})$ serum. The choice of species from which serum is obtained, here and in the antibody solutions, must be selected carefully according to the antibody being used (see Note 3)

7. Heparin stock solution: $\mathrm{ddH}_{2} \mathrm{O}$ with $1 \%(\mathrm{w} / \mathrm{v})$ heparin sodium salt. We use heparin derived from porcine intestinal mucosa

8. Methanol series: $\mathrm{ddH}_{2} \mathrm{O}$ with $25 \%, 50 \%$ or $75 \%$ (v/v) methanol

9. Permeabilisation solution: PTx.2 with $20 \%$ (v/v) DMSO and $2.3 \%$ (w/v) glycine

10. Primary / secondary antibody solution: PTwH with 5\% (v/v) DMSO, 3\% (v/v) serum and appropriate concentration of primary or secondary antibody (see Notes $\mathbf{3}$ )

\subsubsection{Equipment}

1. Plastic and / or glass Pasteur pipettes

2. $2 \mathrm{ml}, 1.5 \mathrm{ml}$ and / or $0.5 \mathrm{ml}$ microcentrifuge tubes

3. Blunt forceps

4. Carousel / rotator

5. Aluminium foil 


\subsection{Refractive index matching reagents and equipment}

\subsubsection{Reagents}

1. BABB: Mix benzyl alcohol (BA) and benzyl benzoate (BB) in a 1:2 ratio (see Note 4)

2. BABB:M: Mix BABB and methanol in a 1:1 ratio

\subsubsection{Equipment}

1. Plastic and / or glass Pasteur pipettes

2. Blunt forceps

3. FluoroDish Cell Culture Dish. There are different diameters available, for small kidneys we have found a $35 \mathrm{~mm}$ diameter to be suitable (see Note 5)

4. Large microscope slides and coverglasses. We use $102 \mathrm{~mm} \times 76 \mathrm{~mm}$ Large Glass Slides and 76mm x 83mm Large Cover Glasses (thickness $=0.19-0.25 \mathrm{~mm}$ )

5. FKM Viton O-Rings, (Internal Diameter: 30mm, Cross Section: 1.5mm). These are resistant to $\mathrm{BA}$ and $\mathrm{BB}$ (see Note 6 )

\subsection{Confocal imaging with single or dual photon excitation}

The confocal microscopy equipment will vary from institute to institute. As such, the individual setup should be tailored with the available resources for the desired application. In our department, we hold an Zeiss LSM 710 Inverted Confocal, with 7 laser lines for excitation $(405,458,488,514,561,594$ and 633nm) and the following objectives: 10x / numerical aperture (NA) 0.45 (working distance $2000 \mu \mathrm{m}$ ), 20x / NA 0.8 Plan Apochromat (working distance $550 \mu \mathrm{m}$ ) and a 25x / NA 0.8 multi-immersion corrected (oil, glycerol or water) Plan Apochromat (working distance $570 \mu \mathrm{m}$ ). However, because of the longer working distances achievable with high NA water immersion objectives, for this application we use a Zeiss LSM 880 Upright Confocal Multiphoton. This has the same 7 laser lines, but also has a SpectraPhysics Mai Tai eHP DeepSee multiphoton laser (690-1040nm). Here, we use 10x / NA 0.5 or 20x / NA 1.0 W-Plan Apochromat Water dipping objectives, with working distances of $3.7 \mathrm{~mm}$ and $2.4 \mathrm{~mm}$ respectively. The latter confocal also has gallium arsenide phosphide (GaAsP) internal and external detectors for high sensitivity, making this confocal excellent for deep tissue imaging. We recommend that the user contact their department's microscopy manager to optimise a setup for deep imaging of optically cleared tissue. 


\subsection{Paraffin embedding}

\subsubsection{Reagents}

1. Ethanol series: $\mathrm{ddH}_{2} \mathrm{O}$ with $70 \%, 85 \%$ or $95 \%$ (v/v) ethanol

2. Histo-Clear II (National Diagnostics)

3. Pelleted Paraffin

\subsubsection{Equipment}

1. Embedding Workstation and Laboratory Oven

2. Glass Vials

3. Embedding Cassettes 


\section{METHODS}

\subsection{Wholemount immunofluorescence}

This protocol assumes that renal tissue has already been isolated and collected. Our laboratory uses embryonic and adult mouse and human fetal kidneys. Ensure the appropriate ethical regulations are followed regarding the handling and sacrifice of animals of the acquisition of human material (see Note 7). All incubation steps are performed in microcentrifuge tubes using a rotator, either at $\mathrm{RT}$ or $4^{\circ} \mathrm{C}$. The precise timings of incubations should be optimised by the user for the desired application or tissue type. Between stages, transfer kidneys carefully between microcentrifuge tubes using equipment dependant on the size of the kidney (see Note 8 ). If using the same microcentrifuge tube between different stages, carefully remove the previous solution using glass or plastic Pasteur pipettes, prior to adding the next solution, ensuring minimal contact with the tissue.

\subsubsection{Fixation and storage}

1. After isolation, rinse kidneys 2-3 times briefly in $\mathrm{ddH}_{2} \mathrm{O}$ prior to fixation to remove residual blood.

2. Incubate renal tissue in $4 \% \mathrm{PFA}$ at $4^{\circ} \mathrm{C}$ on a rotator for an appropriate duration of time. For mouse embryonic kidneys and slices of adult kidney, this can be performed overnight. For larger whole adult kidneys up to three days may be required.

3. Rinse renal tissue in 2-3 changes of PBS, for 5 minutes each at RT. At this stage, tissue can be stored for several months until required.

\subsubsection{Bleaching and permeabilisation}

1. Collect kidneys in $1.5 \mathrm{ml}$ microcentrifuge tubes. Incubate kidneys in increasing concentrations of methanol with $\mathrm{ddH}_{2} \mathrm{O}$ at RT on a shaker. The following concentrations of methanol should be applied for 15-30 minutes each: 25\%, 50\%, $75 \%, 100 \%, 100 \%$.

2. Bleach kidneys by incubation in methanol with $5 \% \mathrm{H}_{2} \mathrm{O}_{2}$ for 2 hours at $4^{\circ} \mathrm{C}$. This reduces autofluorescence; particularly applicable when performing wholemount immunofluorescence of renal tissue due to the strong autofluorescence from the renal vasculature and tubules. For large kidneys, such as mouse adult or human fetal, this step can be performed overnight. 
3. Incubate kidneys in decreasing concentrations of methanol with $\mathrm{ddH}_{2} \mathrm{O}$ at RT on a shaker, in reverse of step 1, with each incubation lasting for 15-30 minutes as follows: $75 \%, 50 \%, 25 \%$.

4. Incubate kidneys in $1 \times \mathrm{PBS}$ at RT for 30 minutes at RT. This is followed by two incubations in PTx.2 for 30 minutes per step at RT.

5. Permeabilise kidneys overnight at $4^{\circ} \mathrm{C}$ by incubation in Permeabilisation solution. The high concentration of DMSO in this solution permeabilises tissue and enables penetration and distribution of subsequent reagents throughout the kidneys.

\subsubsection{Blocking and antibody incubation}

1. Remove kidneys from Permeabilisation solution and incubate in blocking solution for the one day at RT.

2. Incubate kidneys in primary antibody solution at $4^{\circ} \mathrm{C}$ (see Note 9), with appropriate dilution of antibody for 1-3 days, dependant on size of kidney (see Note 10).

3. Remove kidneys from primary antibody solution and incubate 6 times in PTwH at RT for 30 minutes to 1 hour per wash, to wash off all unbound primary antibodies. Use fresh PTwH for each incubation.

4. Incubate kidneys in secondary antibody solution at $4{ }^{\circ} \mathrm{C}$, with appropriate dilution of antibody overnight (see Note 10).

5. Remove kidneys from secondary antibody solution and incubate 6 times in PTwH at RT for 30 minutes to 1 hour per wash, to wash off all unbound secondary antibodies Use fresh PTwH for each incubation.

\subsection{Refractive index matching and preparation for imaging}

During this step, the kidneys are dehydrated, followed by RI matching. For solvent-based, anhydrous RI solutions, this involves dehydration, prior to immersion in solutions such as BABB. Care should be taken during these steps, as solvent based chemicals are often toxic. Preparation should be clean, as solvent-based solutions can cause considerable damage to confocal objectives (see Note 4).

\subsubsection{Dehydration}

1. Dehydrate stained kidneys through a series of increasing methanol concentrations as in Step 3.1.2, at RT with 15-30 minutes each per incubation: $25 \%, 50 \%, 75 \%, 100 \%$.

\subsubsection{Tissue clearing}


1. Place the following reagents and equipment in a fume hood: BABB, BABB:M, glass Pasteur pipettes, FluoroDish, large glass slide and coverslip, BABB resistant rubber ring, glass container filled with methanol. See Table 1 for safety considerations when working with $\mathrm{BABB}$.

2. The following steps vary dependant on the size of the kidney. Step 2 describes setup for a small embryonic kidney. In our hands, this setup permits volume imaging of a maximum sample thickness of approximately $1 \mathrm{~mm}$ (Figure 2a).

a. For small embryonic kidneys, place a prepared kidney, using glass Pasteur pipette, centrally in a FluoroDish, and remove excess methanol (see Note 11).

b. Carefully drop BABB:M solution onto the kidney using a new glass Pasteur pipette, until the kidney is immersed. For small embryonic kidneys this requires a few drops. Leave to clear for 5 minutes, or until the kidney is partially transparent.

c. Carefully remove the BABB:M solution with a glass Pasteur pipette, and dispose of the solution in a glass waste container. Ensure there is no BABB:M solution remaining.

d. Add 1-2 drops of BABB on top of the kidney. The kidney will quickly turn transparent. When adding BABB solution, ensure that the solution is not close to the edges of the FlouroDish.

3. Step 3 describes the setup for larger mouse embryonic, mouse adult or human fetal kidneys. In our hands, this setup permits volume imaging of a maximum sample thickness of approximately $3 \mathrm{~mm}$ (Figures $\mathbf{2 a}$ and $\mathbf{2 b}$ ).

a. Using blunt forceps, place a larger kidney in a BABB:M solution in a small glass container. Leave for 10 minutes, or until the kidney has sunk to the bottom of the container.

b. Remove the kidney with clean blunt forceps and transfer to the middle of a large glass slide. Remove excess BABB:M and place an O-Ring around the kidney.

c. Carefully place several drops of BABB onto the surface of the kidney, until it is immersed. The surface tension of the BABB holds the solution in a 'mound' around the kidney.

d. Place a large glass coverslip down upon the O-Ring. With one finger, gently press the centre of the coverslip until it 'catches' the BABB solution. The tension between the BABB solution and the coverslip forms a 'circular 
bridge', within which the kidney sits. Leave the BABB to render the kidney transparent for one hour. For larger kidneys this may require longer.

\subsection{Confocal imaging with single or two-photon excitation}

The optically cleared kidneys are now ready for imaging with a confocal microscope. The setup for inverted confocal microscopy is relatively straightforward, so here we describe the setup for our Zeiss LSM 880 Upright Confocal Multiphoton microscope. The individual set up for different microscopes including Zeiss, Leica, Nikon and Olympus varies, however the underlying principles remain the same (see Note 12).

\subsubsection{Preparation and setup for upright confocal imaging}

1. Switch on the confocal workstation as per manufacturer instructions (see Note 13).

2. Ensure objective parfocality is set up, so the focus is correct when switching between objectives.

3. Select and insert microscope stage appropriate for used sample preparations described in Step 3.2.2.

4. Adjust height of microscope stage appropriate for sample preparation (see Note 14).

5. Insert sample, with caution (Figure 3a). If a FluoroDish is being used, replace the lid and quickly invert the FluoroDish so that the BABB droplet containing the kidney is hanging from the uppermost surface of glass (see Note 15).

6. Carefully focus sample using eyepieces using 10x dry objective (Figure 3b). This step is an approximation of subsequent focussing using a water immersion lens. Move the stage till kidney is in view and its edges are sharp (see Note 16).

7. Remove objective from focussed position and, using a dropper, place a generous drop of $\mathrm{ddH}_{2} \mathrm{O}$ on the FluoroDish or large glass coverslip (Figure 3c).

8. Replace objective with 10x water immersion objective and return the objective to the focussed position slowly, such that the lens is immersed in the drop of water above the level of, but in line with the BABB-cleared kidney (see Note 17 and Figure 3d).

\subsubsection{Confocal image acquisition with single photon excitation}

1. Turn on Acquisition Mode in software.

2. Setup confocal with appropriate configuration. The Zeiss software has a Smart Manager which automatically turns on appropriate lasers dependant on the fluorophores you input into the software (see Note 18 and Figure 4). 
3. Turn laser powers down, typically to $0.5 \%$ for standard photomultiplier tubes (PMT) and $0.05 \%$ for GaAsP PMT channels.

4. Choose an appropriate track switching strategy. We prefer line switching to visualise all tracks simultaneously during image capture, but frame switching may be more appropriate in some circumstances.

5. Change 'Airy Units' (AU) such that the channel of the longest wavelength corresponds to 1 AU (see Note 19).

6. Click 'Live' then turn on the Range Indicator. The sample may or may not appear in the acquisition window, as black or grey against the blue background.

7. Adjust laser power and detector gain such that structures in the sample are clearly displayed. Watch the image histogram and ensure that the maximum fluorescence intensity is not beyond the detectors range (see Note 20).

8. Turn off Range Indicator and activate the software's z-stack function. Carefully focus through the sample, noting regions of interest, and indicating the top and the bottom of the sample in the z-stack window.

9. Each objective and wavelength has an optimal z-slice thickness. Select the optimal zslice thickness for your set up. For our 10x water immersion objective, this is approximately $3 \mu \mathrm{m}$. This corresponds to the depth of each optical section in the zaxis (see Note 21).

10. Adjust the resolution of the image, the scanning speed, the degree of averaging and the (bi-)directionality of the laser as required (see Note 22).

11. When ready, click 'Start Experiment'. The scan takes time to complete. With a resolution of 512 x 512 pixels scanning takes approximately five minutes for an embryonic day 14.5 mouse kidney. For larger kidneys, such as those form an embryonic day 18.5 mouse, this takes up 15-20 minutes.

12. Remove sample, turn off lasers, and shut down microscope as per instructions provided by microscope manufacturer (see Note 13).

\subsubsection{Confocal image acquisition with two-photon excitation}

1. Turn on Acquisition Mode in software. Ensure the lid is securely placed on the imaging chamber to avoid any outside light hitting the detector (see Note 23).

2. Manually change mirror above imaging chamber such that the two-photon mirror is in place.

3. Setup confocal with two-photon configuration. Wait for the two-photon laser to heat up and switch on. Ideally the laser should run for 1 hour before use. 
4. Tune the laser to the appropriate wavelength [21], we use a single track acquisition with $800 \mathrm{~nm}$ for most fluorophores. Turn laser power down to $0.2 \%$, switch on Range Indicator and Click 'Live', increase the Gain of each detector to locate sample as described in Step 3.3.2.

5. Proceed as per instructions 6-11 of Step 3.3.2.

6. Perform post-image corrections if required (see Note 24). An example of a processed image is shown in Figure 5.

\subsection{Paraffin Embedding}

Here, we describe the processing of renal tissue previously imaged using the confocal for traditional histology and immunohistochemistry. After rehydration in a methanol series, the ethanol dehydration, clearing and paraffin embedding of the kidney is performed as standard. The length and repetition of these stages is particularly important for wax penetration, to make subsequent sectioning as easy as possible.

\subsubsection{Paraffin embedding}

1. Immerse the BABB-cleared kidney in $100 \%$ methanol. For small kidneys, use a plastic Pasteur pipette to fill the FluoroDish with methanol, wait for the kidney to become visible, before transferring the kidney using a glass Pasteur pipette into a $1.5 \mathrm{ml}$ microcentrifuge tube containing $100 \%$ methanol. For larger kidneys, remove the coverslip and use blunt forceps to transfer the kidney into a $1.5 \mathrm{ml}$ microcentrifuge tube containing $100 \%$ methanol (see Note 25). Leave on a rotator at RT for 15-30 minutes.

2. Take the tissue through a series of decreasing methanol concentrations, diluted in water, down to PBS as follows, with 15-30 minutes per step, on a rotator at RT: 75\%, $50 \%, 25 \%$, PBS, PBS.

3. Incubate the kidneys in $70 \%$ ethanol in water at RT. At this stage, tissue can be stored at $4^{\circ} \mathrm{C}$ up to one month.

4. Take the tissue through a series of increasing ethanol concentrations, diluted in water, with 1 hour per step, on a rotator at RT as follows: $85 \%, 95 \%, 100 \%, 100 \%$

5. Transfer the dehydrated kidneys to a glass vial, and fill with Histo-Clear II. Leave to incubate at RT for 1 hour 
6. Remove Histo-Clear II and replace with a 1:1 Histo-Clear II:liquid paraffin solution, and leave in an oven at $70^{\circ} \mathrm{C}$ for 1 hour

7. Remove 1:1 Histo-Clear II:liquid paraffin solution and replace with liquid paraffin, and leave in an oven at $70^{\circ} \mathrm{C}$ for 30 minutes.

8. Repeat liquid paraffin washes as above until 5-6 washes are complete, then embed kidneys as per performed routinely. Leave paraffin to harden overnight at RT.

9. Thereafter, perform sectioning, staining and microscopy as performed routinely (see Note 26). See Figure 6 for an example of a confocal sample processed for histology and immunohistochemistry. 


\section{NOTES}

1. This is by no means an exhaustive list of all the safety information for reagents used in the protocol. Rather, this is a guide for users unfamiliar to the protocol and its reagents. Full safety information should be sought out via the local Control of Substances Hazardous to Health (COSHH) database, online, or through direction from your laboratory manager.

2. The iDISCO website is excellent, providing regular updates to the experimental protocol, a list of validated antibodies, useful tools such as guidance on threedimensional analysis packages, a question and answer and troubleshooting section. For the protocol presented in this Chapter, we have modified and omitted certain steps. For example, we observed good immunolabelling at incubation times shorter than previously described, and removed steps using dichloromethane and dibenzyl ether. The website is accessible at: https://idisco.info.

3. The choice of serum, primary and secondary antibody is crucial, and can be both tricky and frustrating to get right. The below guidance should be taken into consideration, and careful planning is required prior to starting each experiment, as antibodies are costly and the volume of each antibody solution required for this protocol is quite substantial, ranging from $500 \mu \mathrm{m}$ for the smallest samples to 1-2 $\mathrm{ml}$ for larger ones. When a particular antibody combination works well, each reagent should be noted down, in particular the catalogue numbers of the primary and secondary antibodies. It is preferable that the serum should be raised in the same species as the secondary antibody i.e.: donkey serum with donkey secondary antibodies, but this is not always achievable. This can be avoided if the secondary antibodies are pre-adsorbed against the serum chosen for the experiment. Primary antibodies which work in other applications do not necessarily work in wholemount immunofluorescence, so the user will likely have to look at previous publications carefully, and trial multiple antibodies with the same target to identify the best for this application. Many antibodies are not compatible with the methanol treatment and dehydration used in this protocol, but the original iDISCO protocol suggests incubation of PFA-fixed frozen tissue sections with or without methanol, prior to immunolabelling, to determine methanol compatibility. Fluorescent secondary antibodies must be selected with specificity for the primary antibodies in mind and also excitation and emission spectra. An example of a good choice of secondary 
conjugates for immunofluorescence would be 488, 546 and 633. In contrast, a 594 and 633 combination would cause considerable overlap between the spectra, that may require post-imaging corrections. We tend to use Alexa Fluor secondary antibodies due to their good signal and photostability, the latter being important when imaging large volumes to avoid bleaching during image acquisition.

4. BABB is an extremely toxic material and should be handled with the utmost caution. Due to the solvent properties of $\mathrm{BABB}$, it readily dissolves adhesives, such as those used to construct microscope objectives. It is for this reason that BABB should always be enclosed, and never come into contact with the objective or lens. The experimental setup provided in this Chapter is a good solution, but improper handling of the sample, or lack of concentration when adjusting the focus of the confocal, are extremely costly errors. Leica Microsystems have also developed an 20x objective (NA, 0.95) which is designed for immersion in $\mathrm{BABB}$, with a modest working distance of $1.95 \mathrm{~mm}$. In any case, contact your confocal microscope manager prior to using $\mathrm{BABB}$, and we recommend that the first few tries of this protocol should be performed under supervision.

5. The FluoroDish has a $0.17 \mathrm{~mm}$ thick coverslip base, which permits imaging from underneath the sample using an inverted confocal, or inversion of the dish and imaging using an upright confocal.

6. Screening for compounds which are resistant to BABB, we found that FKM Viton has minimal chemical reactivity with BA and BB. FKM Viton rings, known as O-Rings, can be purchased in a range of diameters and thicknesses. We recommend an internal diameter of $30 \mathrm{~mm}$ such that BABB does not come into contact with the edges of the ring. The cross sectional size depends upon the size of the sample. Ideally, the sample should be as close to the coverslip as possible. For samples up to $1 \mathrm{~mm}$ in height, a $1 \mathrm{~mm}$ thick O-Ring is suitable, as samples shrink during to methanol dehydration. We have rings with depths from 0.5 to $3.5 \mathrm{~mm}$ in $0.5 \mathrm{~mm}$ increments. As our 10x / NA0.5 W-Plan Apochromat objective has a working distance of $3.7 \mathrm{~mm}$, we can confidently image to the bottom of any sample in any of these O-Rings without the objective contacting the uppermost coverslip. For our 20x / NA1.0 W-Plan Apochromat with a working distance of $2.4 \mathrm{~mm}$, we only use O-Rings up to $2 \mathrm{~mm}$ deep. A potential source of such O-Rings is Polymax.

7. Animals should be sacrificed in accordance with national legislation. We have access to human fetal material through the Human Developmental Biology Resource, a 
United Kingdom-based resource for which any laboratory can apply to obtain material [22]. Clinical material and patient samples should be acquired with the relevant ethical approval. We have not tried this protocol with clinical material, but if optimised for this application, has the potential to become a powerful tool for probing disease processes in human samples.

8. For small mouse embryonic kidneys we use a glass or plastic Pasteur pipette. Transfers of larger embryonic or adult mouse, or human fetal kidneys, are performed carefully with use of a blunt forceps, working quickly to minimise the time that the samples are exposed to air, as drying of the samples can lead to artefact in imaging.

9. The original iDISCO+ protocol recommends several steps including permeabilisation, blocking and antibody incubation be performed at $37^{\circ} \mathrm{C}$. However we only have Carousels at $4^{\circ} \mathrm{C}$ or RT. There is evidence to suggest that increasing the temperature of incubation decreases the specificity of the antibody, increasing non-specific binding $[23,24]$.

10. The concentration of antibody required for each experiment varies considerably. We find that most suitable primary antibodies for our applications appear to work well at a concentration of 1:100. However some work better at 1:50, and others work best at lower concentrations of 1:200 or 1:500. We recommend starting at 1:100 and adjusting based on the signal to background achieved in the final image. We use all secondary antibodies at a concentration of 1:200. During antibody incubation stages, we transfer small kidneys into a $500 \mu \mathrm{l}$ microcentrifuge tube to minimise the volume of antibody used and avert the cost, and transfer samples back to larger $1.5 \mathrm{ml}$ tubes after incubation is complete. This may not be achievable for larger samples. After the secondary antibody is applied, protect the samples from light by covering the tubes in aluminium foil.

11. At this stage, we recommend labelling the FluoroDish or glass slide with permanent marker, indicating details of the experiment, repeat number and so on. After the protocol is completed and the kidney is virtually invisible, it becomes impossible to distinguish one sample from another.

12. Confocal microscopy can be relatively complicated for the uninitiated. Though incredible, journal cover-worthy images can be generated, it is easy to get wrong, and carelessness and lack of understanding of the principles of confocal can lead to poor image quality or interpretation, or damage to very expensive equipment. Instead of relying on the microscopy manager, we recommend that all users familiarise 
themselves with the concepts of confocal microscopy using the wealth of online resources available. One of our favourites is the Nikon MicroscopyU website, providing simple but detailed explanations of several topics in confocal and twophoton microscopy: https://www.microscopyu.com/techniques/confocal.

13. For our confocal microscope, setting up the system involves turning on the switches to the microscope and laser power supply, workstation computer and components computer in a particular sequence, after which the fluorescence lamp, Zen software and lasers are switched on. There is also an appropriate sequence of instructions to shut down the microscope, to avoid damage to the system. Of note, some lasers, such as the 488 Argon laser, require several minutes to cool prior to shutting down. Consult your departmental microscopy expert or the microscope manufacturer for full details.

14. We always start with a 10x air objective, which is in focus when $5 \mathrm{~mm}$ above the coverslip or FluoroDish. This is used for both estimating the correct height to set the microscope stage, and correctly focussing the sample prior to switching to water immersion lenses. Initial focussing with water immersion objectives not advised as the distance between objective and coverslip is difficult to judge through the water. By setting objective parfocality on a safe (BABB-free) sample, focussing with the simple to use 10x air objective ensures the water immersion objectives will automatically be in the correct focal plane.

15. Inverting the FluoroDish into a 'hanging drop' configuration can be quite challenging. The droplet of BABB has to be contained in the middle of the FluoroDish surface, as contact with the sides will cause the $\mathrm{BABB}$ to react with the adhesive used to construct the dish, forming a precipitate and making the kidney impossible to image. It requires a smooth and quick action to invert the FluoroDish whilst keeping the kidney central, and the high surface tension of BABB prevents the kidney from falling. The largest sample we have performed this successfully with was a whole kidney from an embryonic day 17.5 mouse fetus, bearing in mind that the BABB protocol and methanol dehydration causes the sample to shrink [5]. We advise a few practise runs with a drop of BABB alone. At the end of the imaging session, it is critical to re-invert the FluoroDish back to its original configuration for long term storage.

16. When focussing the sample, a fluorescent bulb is active, so the fluorescent kidney comes into view, enabling alignment of the objective with the sample. 
17. The air objectives on the confocal are changed to water immersion objectives to minimise the effects of RI mismatch. The RI of BABB is approximately 1.56 [1], whereas glass is 1.51 , water 1.33 and air is 1.0 . When imaging samples with RI mismatched objectives, an optical phenomenon termed spherical aberration leads to a loss of resolution and signal strength. As imaging depth increases so does the degree of spherical aberration, making imaging of deep tissue challenging. Most confocal software provides settings that adjust for spherical aberration by increasing the power of the laser as imaging depth increases, to compensate for the loss of signal intensity. However, this does not restore the loss of resolution. Spherical aberration is more apparent with higher NA objectives, so if high resolution is required, (therefore needing high NA) then it is vital to minimise the RI mismatch between BABB and the objective. Ideally imaging would be performed with an objective designed for high RI immersion media, such as an oil (RI 1.51) immersion objective, as this is close to BABB (RI 1.56) and therefore minimises spherical aberration. However, oil immersion objectives have short working distances, with nothing longer than $570 \mu \mathrm{m}$ currently available from Zeiss. Leica have developed a 20x / NA 0.95 BABB objective, designed for RI 1.56, but this is not compatible with Zeiss systems. Long working distance, high NA water immersion objectives are a good compromise, providing high resolution with less aberration than high NA air objectives. While the RIs in this setup are still not matched, we are able to image renal structures down to cellular resolution at depths beyond $1 \mathrm{~mm}$. If detail of subcellular structures, such as mitochondria, are required, using an oil immersion objective would be better. This is at the sacrifice of imaging depth, as such objectives would have shorter working distances, so the renal tissue may have to be coarsely sliced using a vibratome prior to starting the protocol.

18. If using an Argon laser to visualise a 488 fluorophore, wait for five minutes after switching the laser on, as it takes this time to warm up. Ideally argon lasers should be run on standby for an hour before imaging to stabilise the laser.

19. The defining feature of confocal microscopes is the use of a pinhole to ensure that only light from the plane of interest reaches the imaging detector, and out-of-focus light is excluded. Typically we set the pinhole diameter to match $1 \mathrm{AU}$ for the longest wavelength channel, taking the view that axial resolution is determined by the longest wavelength (resolution decreases as wavelength increases). When the multiphoton 
laser is used, the pinhole is unnecessary due to the properties of two-photon excitation.

20. A higher laser power will photobleach the sample more quickly, whereas a higher gain will increase noise, leading to graininess of the image. The optimal image is a balance between these two components. When adjusting these, take into account the relative intensity of the signal to background and the image histogram. If fluorescence intensity is too high then the full range of fluorescence is 'cropped', and this information is lost from the final image.

21. Distance is distorted when imaging into a sample with a different NA than the objective immersion media. The z-stack window has the option of inserting the refractive index ratio of the imaging material and objective immersion media. This then corrects the axial distance accordingly. We do not use this feature, but correct axial distance post-acquisition. Actual distance in the sample is the z-step distance multiplied by (RI Sample / RI Objective). For an air immersion objective using a BABB-cleared sample, a $\mathrm{z}$ step of $1 \mu \mathrm{m}$ gives a distance of $1 \mu \mathrm{m} * 1.56 / 1.00=1.56$ $\mu \mathrm{m}$. The same sample using a z-step of $1 \mu \mathrm{m}$ with a water immersion objective gives $1 \mu \mathrm{m} * 1.56 / 1.33=1.165 \mu \mathrm{m}$.

22. As a principle, higher resolution, slower scanning speed, single directionality and higher pixel averaging all result in better image quality, but increase the time taken to complete scanning. We tend to set the following parameters: resolution, 512 x 512 or 1024 x 1024; speed, 7-9; directionality, bi-directional; averaging, 2. The same settings are applied for two-photon imaging, with a higher pixel averaging of 4 due to more noise with this imaging modality.

23. Two-photon imaging uses non-descanned detectors (no pinhole is present) so must be performed in the dark to prevent detection of ambient light. Exposure of the system to ambient light may damage the detectors.

24. Two-photon excitation harbours several potential advantages over standard confocal imaging for this application. In the context of fluorescence imaging of immunolabelled and cleared kidneys, the laser penetrates the sample better due to a higher wavelength, and captures more signal, given that out-of-focus light (produced as an effect of spherical aberration) is not obstructed by the presence of a pinhole. However, two-photon excitation generates considerably more autofluorescence from structures such as collagen and erythrocytes. This autofluorescence is greater at shorter wavelengths, and is particularly apparent in the green and yellow regions of 
the light spectrum. When imaging with multiple fluorophores, there is a greater chance of bleed through arising between fluorescence channels of the image as twophoton excitation at $800 \mathrm{~nm}$ often excites all fluorophores. Post image corrections to automatically correct for this effect, such as spectral unmixing, may need to be performed as indicated by your local microscopy expert. Given this comparison of single and two-photon excitation, the imaging modality we use depends on the desired application, and is influenced by the range of fluorophores for each experiment.

25. Upon contact with $100 \%$ methanol, the BABB-cleared kidney starts to become white and opaque, which makes subsequent handling and transfers considerably easier.

26. Remarkably, fluorescence is preserved even after paraffin embedding, sectioning and staining. We have omitted further detail of sectioning and staining as these are well established protocols, and will likely be performed slightly differently in each laboratory. The key points here are that the duration of ethanol changes and length and frequency of liquid paraffin stages is critical to ensure satisfactory embedding of the kidney. Combes and colleagues suggest that unmasking using compounds such as citrate buffer is not necessary, as the BABB treatment appears to unmask antigens adequately [19]. We have not determined whether this protocol preserves RNA, but previous data of in situ hybridisation patterns imaged using optical projection tomography appears to suggest that it does. Fluorescent proteins are not preserved by this protocol, however anti-fluorescent protein secondary antibodies may be used, or the protocol may be modified according to newer iterations to combine immunolabelling with detection of fluorescent protein expression $[25,26]$. 


\section{Acknowledgements}

The authors would like to thank Drs Karen Price, Maria Kolatsi-Joannou, Sarah Ivins, Catherine Roberts and the rest of the Kidney Disease and Development Group and the Molecular Medicine Unit at UCL Great Ormond Street Institute of Child Health for their guidance, and the staff of the UCL Confocal Imaging Facility for their advice. We would also like to acknowledge Dr Nicolas Renier (Institut du Cerveau et de la Moelle Epiniere), Dr Alexander Combes and Professor Melissa Little (Murdoch Children's Research Institute) for their email correspondence and clarifications of the methods used in their publications. DJ is supported by funding from the Child Health Research PhD Studentship at UCL Great Ormond Street Institute of Child Health and the UCL MB PhD Programme. DAL's laboratory is supported by a Medical Research Council New Investigator Award (MR/J003638/1) and project grant (MR/P018629/1), project grants from Diabetes UK (13/0004763, 15/0005283) and Kidney Research UK (RP36/2015). under the National Institute for Health Research (NIHR) Biomedical Research Centre (BRC) at Great Ormond Street Hospital (GOSH) for Children NHS Foundation Trust and University College London. Microscopy was performed at the Light Microscopy Core Facility at UCL Great Ormond Street Institute of Child Health, also supported by the NIHR BRC at GOSH (17DD08). 


\section{References}

1. Richardson DS, Lichtman JW (2015) Clarifying Tissue Clearing. Cell 162: 246-257

2. Tainaka K, Kuno A, Kubota SI, Murakami T, Ueda HR (2016) Chemical Principles in Tissue Clearing and Staining Protocols for Whole-Body Cell Profiling. Annu Rev Cell Dev Biol 32: 713-741

3. Puelles VG, Moeller MJ, Bertram JF (2017) We can see clearly now: optical clearing and kidney morphometrics. Curr Opin Nephrol Hypertens 26: 179-186

4. Richardson DS, Lichtman JW (2017) SnapShot: Tissue Clearing. Cell 171: 496-496

5. Hama H, Kurokawa H, Kawano H, Ando R, Shimogori T, Noda H, Fukami K, Sakaue-Sawano A, Miyawaki A (2011) Scale: a chemical approach for fluorescence imaging and reconstruction of transparent mouse brain. Nat Neurosci 14: 1481-1488

6. Hama H, Hioki H, Namiki K, Hoshida T, Kurokawa H, Ishidate F, Kaneko T, Akagi T, Saito T, Saido T, Miyawaki A (2015) ScaleS: an optical clearing palette for biological imaging. Nat Neurosci 18: 1518-1529

7. Kuwajima T, Sitko AA, Bhansali P, Jurgens C, Guido W, Mason C (2013) ClearT: a detergent- and solvent-free clearing method for neuronal and non-neuronal tissue. Development 140: 1364-1368

8. Dodt HU, Leischner U, Schierloh A, Jährling N, Mauch CP, Deininger K, Deussing JM, Eder M, Zieglgänsberger W, Becker K (2007) Ultramicroscopy: three-dimensional visualization of neuronal networks in the whole mouse brain. Nat Methods 4: 331-336

9. Puelles VG, van der Wolde JW, Schulze KE, Short KM, Wong MN, Bensley JG, Cullen-McEwen LA, Caruana G, Hokke SN, Li J, Firth SD, Harper IS, Nikolic-Paterson DJ, Bertram JF (2016) Validation of a Three-Dimensional Method for Counting and Sizing Podocytes in Whole Glomeruli. J Am Soc Nephrol 27: 3093-3104

10. Lindström NO, McMahon JA, Guo J, Tran T, Guo Q, Rutledge E, Parvez RK, Saribekyan G, Schuler RE, Liao C, Kim AD, Abdelhalim A, Ruffins SW, Thornton ME, Basking L, Grubbs B, Kesselman C, McMahon AP (2018) Conserved and Divergent Features of Human and Mouse Kidney Organogenesis. J Am Soc Nephrol 29: 785-805

11. Ertürk A, Becker K, Jährling N, Mauch CP, Hojer CD, Egen JG, Hellal F, Bradke F, Sheng M, Dodt HU (2012) Three-dimensional imaging of solvent-cleared organs using 3DISCO. Nat Protoc 7: 19831995

12. Renier N, Wu Z, Simon DJ, Yang J, Ariel P, Tessier-Lavigne M (2014) iDISCO: a simple, rapid method to immunolabel large tissue samples for volume imaging. Cell 159: 896-910

13. Renier N, Adams EL, Kirst C, Wu Z, Azevedo R, Kohl J, Autry AE, Kadiri L, Umadevi Venkataraju K, Zhou Y, Wang VX, Tang CY, Olsen O, Dulac C, Osten P, Tessier-Lavigne M (2016) Mapping of Brain Activity by Automated Volume Analysis of Immediate Early Genes. Cell 165: 1789-1802

14. de Chaumont F, Dallongeville S, Chenouard N, Hervé N, Pop S, Provoost T, Meas-Yedid V, Pankajakshan P, Lecomte T, Le Montagner Y, Lagache T, Dufour A, Olivo-Marin JC (2012) Icy: an open bioimage informatics platform for extended reproducible research. Nat Methods 9: 690-696

15. Peng H, Bria A, Zhou Z, Iannello G, Long F (2014) Extensible visualization and analysis for multidimensional images using Vaa3D. Nat Protoc 9: 193-208 
16. Royer LA, Weigert M, Günther U, Maghelli N, Jug F, Sbalzarini IF, Myers EW (2015) ClearVolume: open-source live 3D visualization for light-sheet microscopy. Nat Methods 12: 480-481

17. Short K, Hodson M, Smyth I (2013) Spatial mapping and quantification of developmental branching morphogenesis. Development 140: 471-478

18. Klingberg A, Hasenberg A, Ludwig-Portugall I, Medyukhina A, Männ L, Brenzel A, Engel DR, Figge MT, Kurts C, Gunzer M (2017) Fully Automated Evaluation of Total Glomerular Number and Capillary Tuft Size in Nephritic Kidneys Using Lightsheet Microscopy. J Am Soc Nephrol 28: 452-459

19. Combes AN, Short KM, Lefevre J, Hamilton NA, Little MH, Smyth IM (2014) An integrated pipeline for the multidimensional analysis of branching morphogenesis. Nat Protoc 9: 2859-2879

20. Brzóska HL, d'Esposito AM, Kolatsi-Joannou M, Patel V, Igarashi P, Lei Y, Finnell RH, Lythgoe MF, Woolf AS, Papakrivopoulou E, Long DA (2016) Planar cell polarity genes Celsrl and Vangl2 are necessary for kidney growth, differentiation, and rostrocaudal patterning. Kidney Int 90: 1274-1284

21. Mütze J, Iyer V, Macklin JJ, Colonell J, Karsh B, Petrášek Z, Schwille P, Looger LL, Lavis LD, Harris TD (2012) Excitation spectra and brightness optimization of two-photon excited probes. Biophys J 102: 934-944

22. Gerrelli D, Lisgo S, Copp AJ, Lindsay S (2015) Enabling research with human embryonic and fetal tissue resources. Development 142: 3073-3076

23. Güven E, Duus L, Lydolph MC, Jørgensen CS, Laursen I, Houen G (2014) Non-specific binding in solid phase immunoassays for autoantibodies correlates with inflammation markers. J Immunol Methods 403: 26-36

24. Ward JM, Rehg JE (2014) Rodent immunohistochemistry: pitfalls and troubleshooting. Vet Pathol 51: 88-101

25. Schwarz MK, Scherbarth A, Sprengel R, Engelhardt J, Theer P, Giese G (2015) Fluorescent-protein stabilization and high-resolution imaging of cleared, intact mouse brains. PLoS One 10: e0124650 doi: 10.1371/journal.pone.0124650

26. Pan C, Cai R1, Quacquarelli FP, Ghasemigharagoz A, Lourbopoulos A, Matryba P, Plesnila N, Dichgans M, Hellal F, Ertürk A (2016) Shrinkage-mediated imaging of entire organs and organisms using uDISCO. Nat Methods 13:859-867 
TABLES

\section{Table 1: Experimental reagents, storage conditions and safety considerations}

\begin{tabular}{|c|c|c|}
\hline Reagent & Storage & Suggested safety considerations \\
\hline $4 \%$ PFA & $\begin{array}{l}\text { Store at }-20^{\circ} \mathrm{C} \text { for up to one year. We recommend storing in } \\
\text { several small aliquots and defrosting when required. Avoid } \\
\text { repeat freeze-thaw cycles, and once defrosted, store at } 4^{\circ} \mathrm{C} \text { for } \\
\text { not longer than one week }\end{array}$ & $\begin{array}{l}\text { PFA is an extremely hazardous chemical, is absorbed } \\
\text { through the skin and is toxic to mucus membranes. Make } \\
\text { up in fume hood, and take precautions when handling. } \\
\text { Dispose of as per departmental guidelines }\end{array}$ \\
\hline Antibody solution & $\begin{array}{l}\text { Make up fresh, storable for one week at } 4^{\circ} \mathrm{C} \text { Add antibodies } \\
\text { immediately prior to use. For fluorophore-conjugated } \\
\text { antibodies, protect from light with aluminium foil }\end{array}$ & $\begin{array}{l}\text { Sodium azide is toxic, handle with care when making up } \\
\text { PBS stock solution }\end{array}$ \\
\hline BABB & $\begin{array}{l}\text { Store in dark glass bottles, protected and away from light, at RT. } \\
\text { We tend to make this fresh from benzyl alcohol and benzyl } \\
\text { benzoate and store for one month at a time }\end{array}$ & $\begin{array}{l}\text { BABB is toxic. Use with extreme caution under fume } \\
\text { hood. When handling BABB, we recommend keeping a } \\
\text { glass beaker filled with methanol to hand in case of } \\
\text { spillage. BABB dissolves plastic and glue, so must be } \\
\text { kept in glass containers at all times. Dispose of as per } \\
\text { departmental guidelines }\end{array}$ \\
\hline BABB:M & See BABB & See BABB \\
\hline Bleaching solution & Make up fresh, use immediately. Store at $4^{\circ} \mathrm{C}$ for up to one day & $\begin{array}{l}\text { Hydrogen peroxide is corrosive, and skin contact and } \\
\text { exposure to fumes should be minimised }\end{array}$ \\
\hline Blocking solution & Make up fresh, storable for up to one week at $4^{\circ} \mathrm{C}$ & $\begin{array}{l}\text { Sodium azide is toxic, handle with care when making up } \\
\text { PBS stock solution }\end{array}$ \\
\hline Ethanol & Store ethanol series indefinitely at RT & $\begin{array}{l}\text { Take care of flammability, be aware of local Fire and } \\
\text { Safety guidelines }\end{array}$ \\
\hline Histo-Clear II & Store indefinitely at RT & $\begin{array}{l}\text { Take care of flammability, be aware of local Fire and } \\
\text { Safety guidelines. Minimise skin contact and exposure to } \\
\text { fumes. Dispose of as per departmental guidelines }\end{array}$ \\
\hline Methanol & Store methanol series indefinitely at RT & $\begin{array}{l}\text { Methanol is toxic, minimise skin contact and exposure to } \\
\text { fumes. Take care of flammability, be aware of local Fire } \\
\text { and Safety guidelines. Dispose of as per departmental } \\
\text { guidelines. }\end{array}$ \\
\hline Paraffin & Store pellets indefinitely at RT & $\begin{array}{l}\text { Take care when handling liquid paraffin, may cause } \\
\text { burns }\end{array}$ \\
\hline $\begin{array}{l}\text { Permeabilisation } \\
\text { solution }\end{array}$ & $\begin{array}{l}\text { If made up using } 1 \times \text { PBS with } 0.02 \% \text { sodium azide, this can be } \\
\text { stored at RT for up to one month }\end{array}$ & $\begin{array}{l}\text { Take care when handling due to high concentration of } \\
\text { DMSO, which is readily absorbed into the skin and can } \\
\text { carry toxic materials into the body. Avoid skin contact }\end{array}$ \\
\hline $1 \mathrm{x}$ PBS & $\begin{array}{l}\text { If made up using } 0.02 \% \text { sodium azide, this can be stored at RT } \\
\text { for up to one month }\end{array}$ & $\begin{array}{l}\text { Sodium azide is toxic, handle with care when making up } \\
\text { PBS stock solution }\end{array}$ \\
\hline PTwH & $\begin{array}{l}\text { If made up using } 1 \times \text { PBS with } 0.02 \% \text { sodium azide, this can be } \\
\text { stored at RT for up to one month }\end{array}$ & $\begin{array}{l}\text { Sodium azide is toxic, handle with care when making up } \\
\text { PBS stock solution }\end{array}$ \\
\hline PTx.2 & $\begin{array}{l}\text { If made up using } 1 \times \text { PBS with } 0.02 \% \text { sodium azide, this can be } \\
\text { stored at RT for up to one month }\end{array}$ & $\begin{array}{l}\text { Sodium azide is toxic, handle with care when making up } \\
\text { PBS stock solution }\end{array}$ \\
\hline
\end{tabular}




\section{FIGURES}

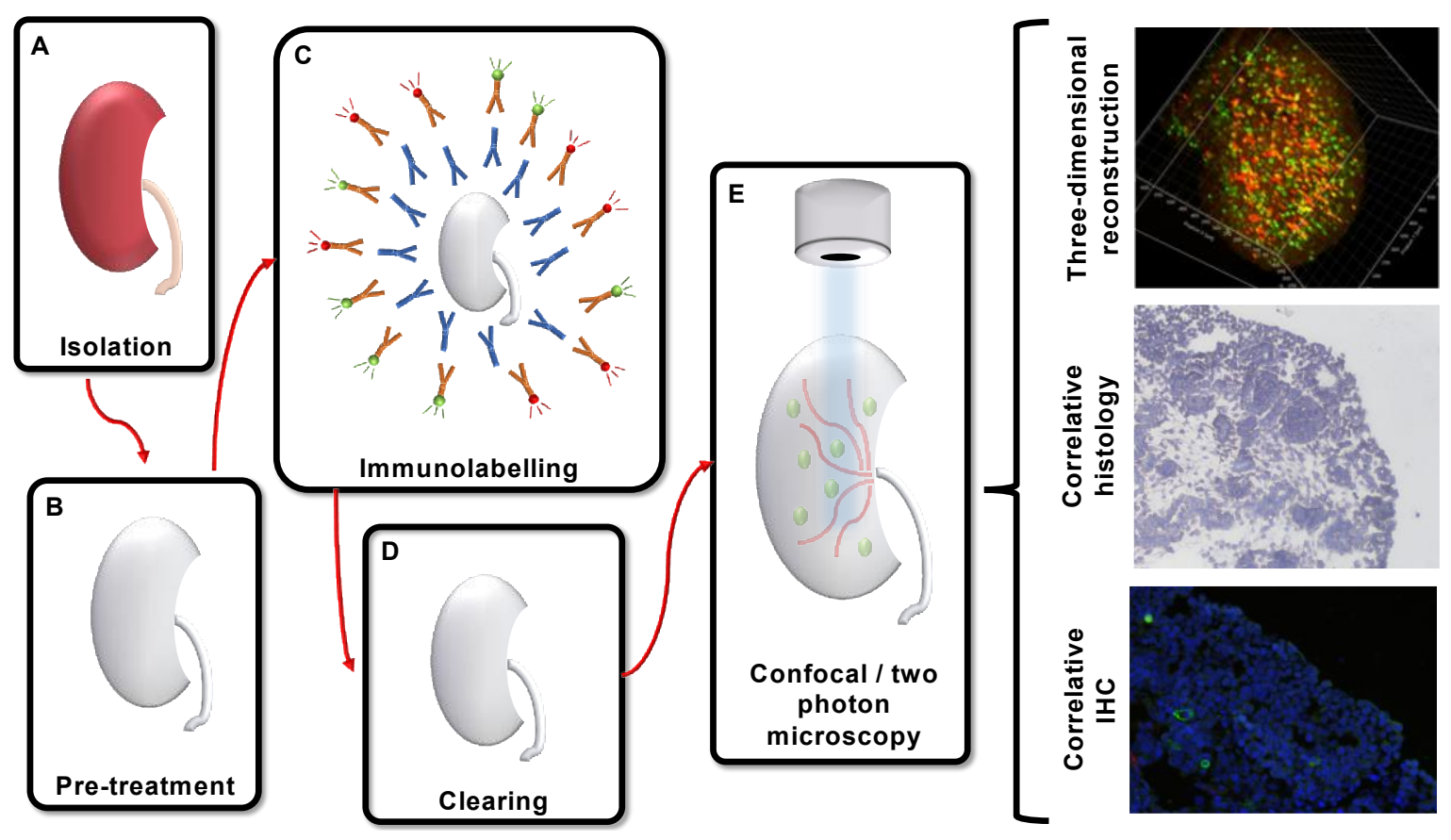

Figure 1. Workflow for deep imaging of renal tissue. A) Isolation of the mouse or human renal material B) applying compounds such as DMSO to permeabilise, and hydrogen peroxide to bleach C) Immunolabelling using direct or indirect immunohistochemistry D) Clearing using solvent based solutions, such as BABB E) Imaging using confocal microscopy with or without two-photon excitation. With this protocol, optional paraffin embedding and sectioning can be performed after confocal imaging to correlatively analyse confocal findings, by probing histology for disease phenotypes, or further characterising confocal imaging using traditional fluorescence IHC. IHC, immunohistochemistry. 


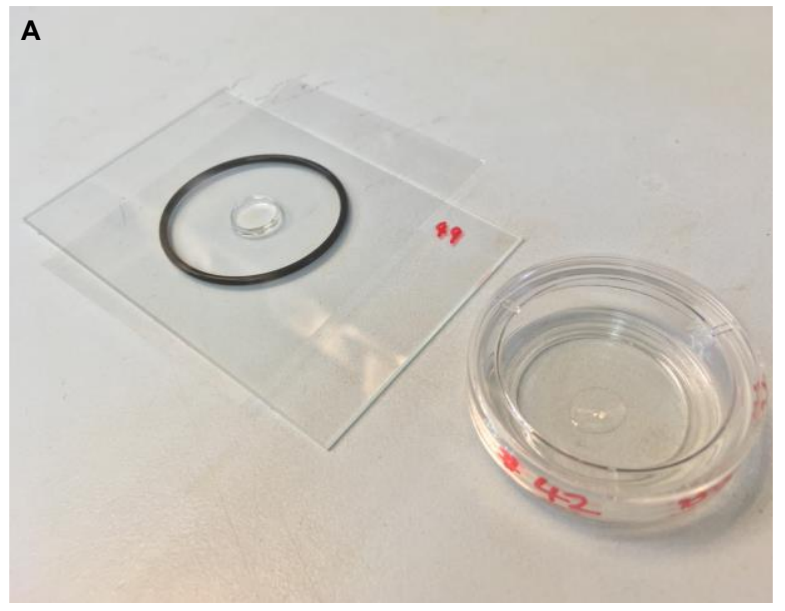

B

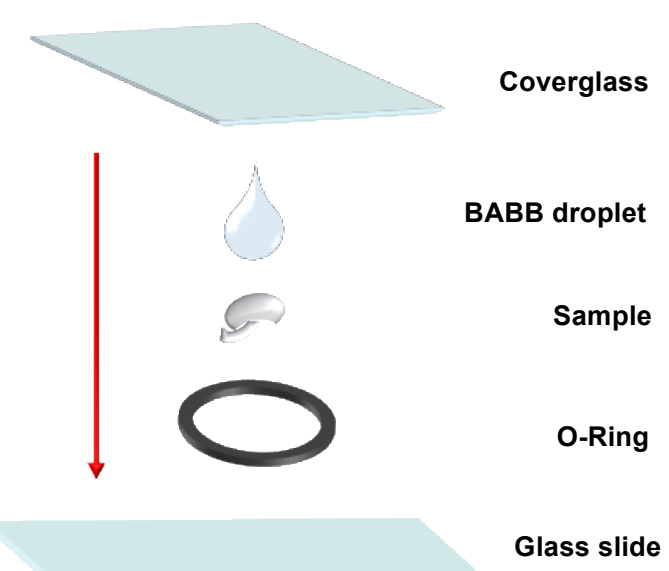

Figure 2. Set up for upright confocal imaging of BABB-cleared tissue. A) FluoroDish setup for small renal material (right), large coverglass and O-Ring configuration for larger renal material (left). B) The individual components of the coverglass / O-Ring setup. Each component is applied in sequence, starting with the glass slide and finishing with the coverglass. 

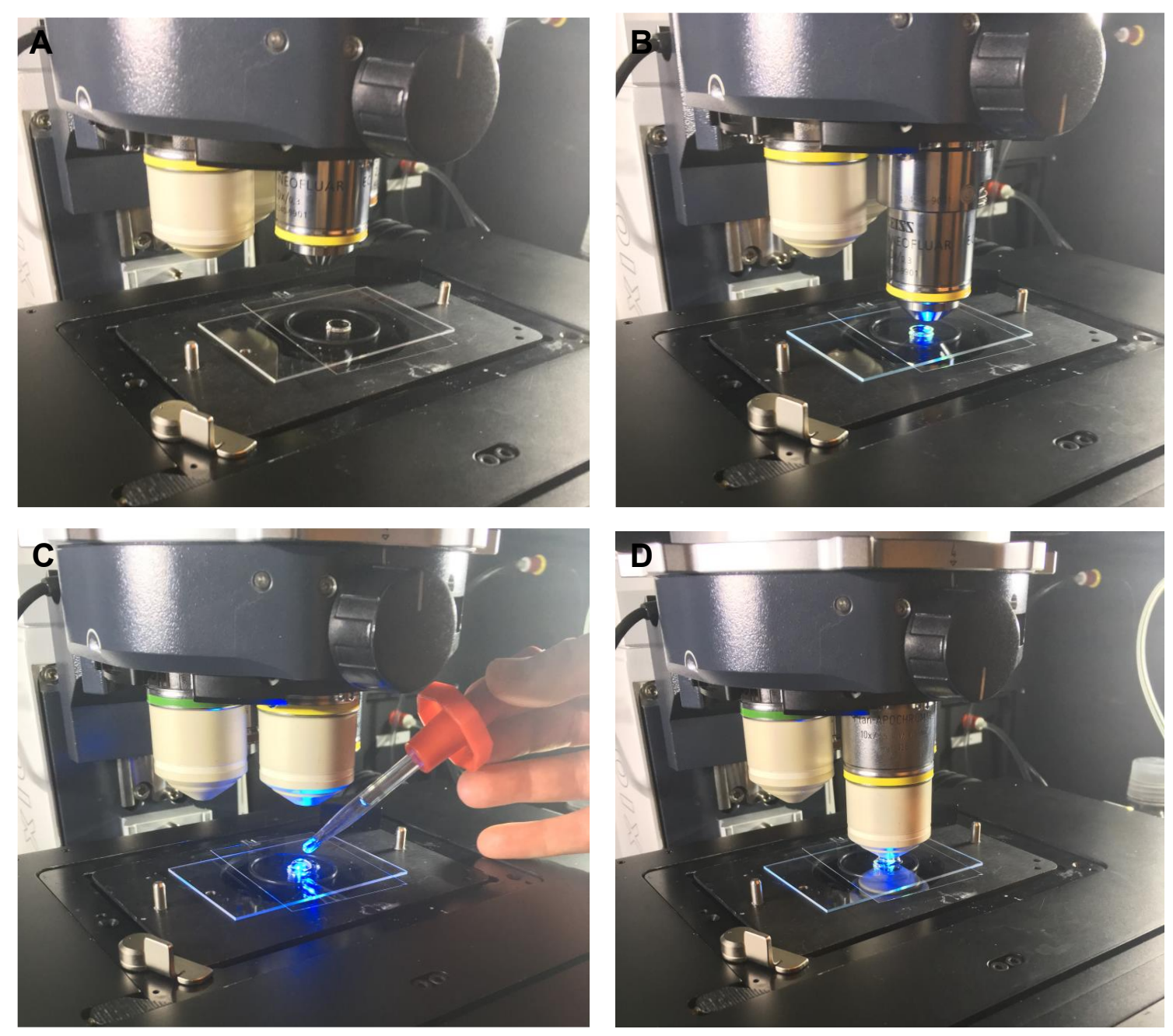

Figure 3. Setting up and focusing the upright confocal objectives for imaging BABB-

cleared tissue. A) After placing the appropriate holder in the microscope stage and adjusting its height, carefully place the sample underneath a low-resolution air immersion objective. B) Place the objective in the focused position and locate sample using eyepiece. C) Remove objective from focused position and place a generous drop of distilled water on the coverglass, below which the BABB and the sample rest. D) Change to a water immersion objective and carefully lower into the focused position, bringing the sample into clear view with sharp edges. 


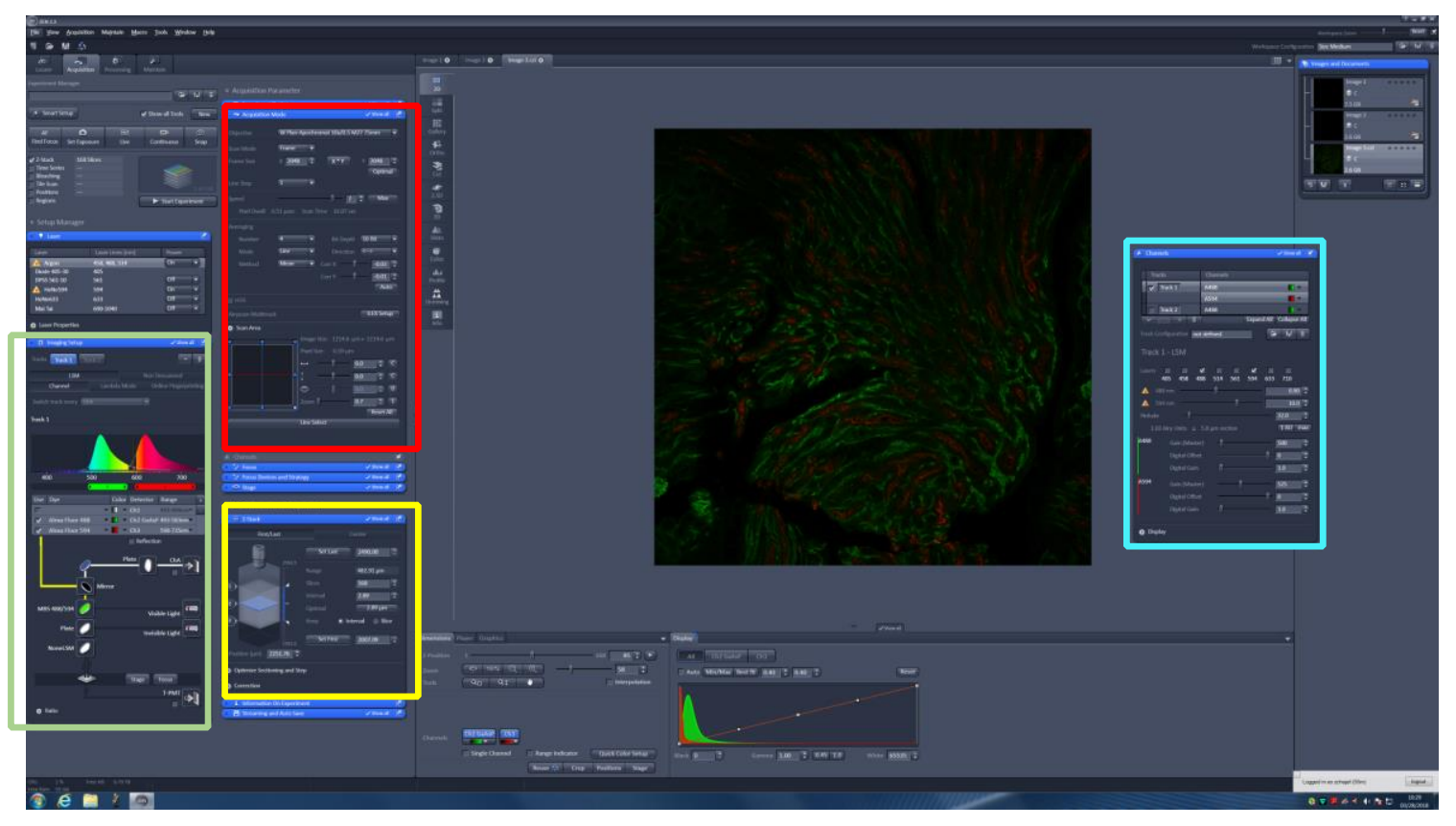

Figure 4. Screenshot of the Zen confocal software. The Acquisition Mode, including dimensions, speed, pixel averaging and scan area is boxed in red. The Imaging Setup, above which the laser switches sit, and within which the settings for the detectors and mirror sit is boxed in green. The Z-Stack function, which must be activated by ticking the box in the top left of the screen, and with which the optical sectioning depth and bottom and top of the sample can be registered, is boxed in yellow. The Channels tool, with which laser powers, gain and airy units can be set, is boxed in light blue. These tools and windows can be configured as the user prefers. 

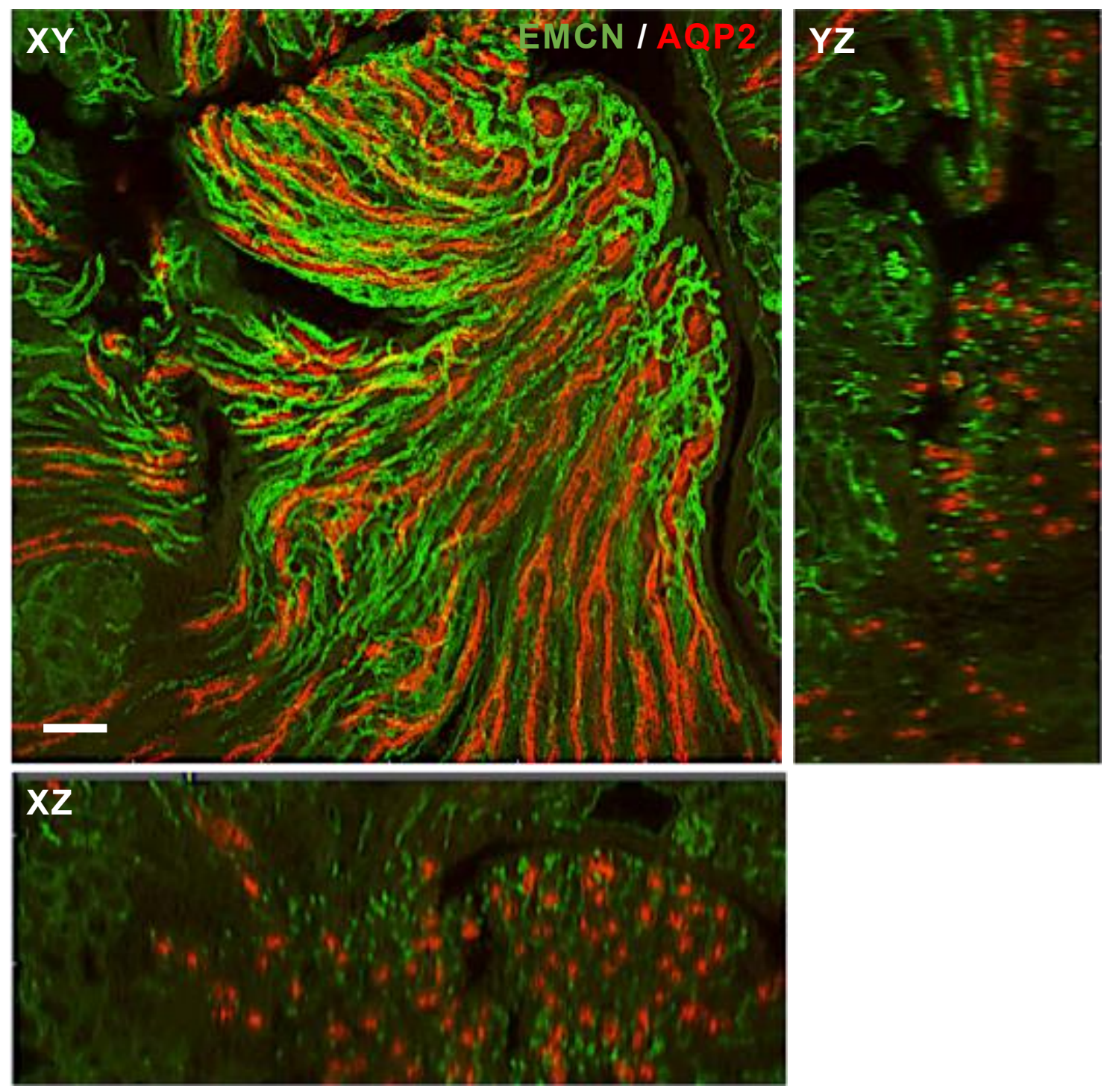

Figure 5. Example of confocal imagery. The image is taken from an embryonic day 18.5 kidney, stained with rat anti-mouse endomucin (EMCN; 1:50; Santa-Cruz; sc-53941) and rabbit anti-mouse aquaporin 2 (AQP2; 1:50; Abcam; ab109926). A maximum intensity projection is shown, to display the continuity of EMCN+ blood vessels and AQP2+ collecting ducts. Orthogonal slices of the confocal scan is shown, letters in the top left of each image correspond to the plane of the image. The z-thickness of the optical section is 29 $\mu \mathrm{m}$, and the orientation of optical sectioning is indicated using the white and yellow crosshairs. Scale bar, $200 \mu \mathrm{m}$. 


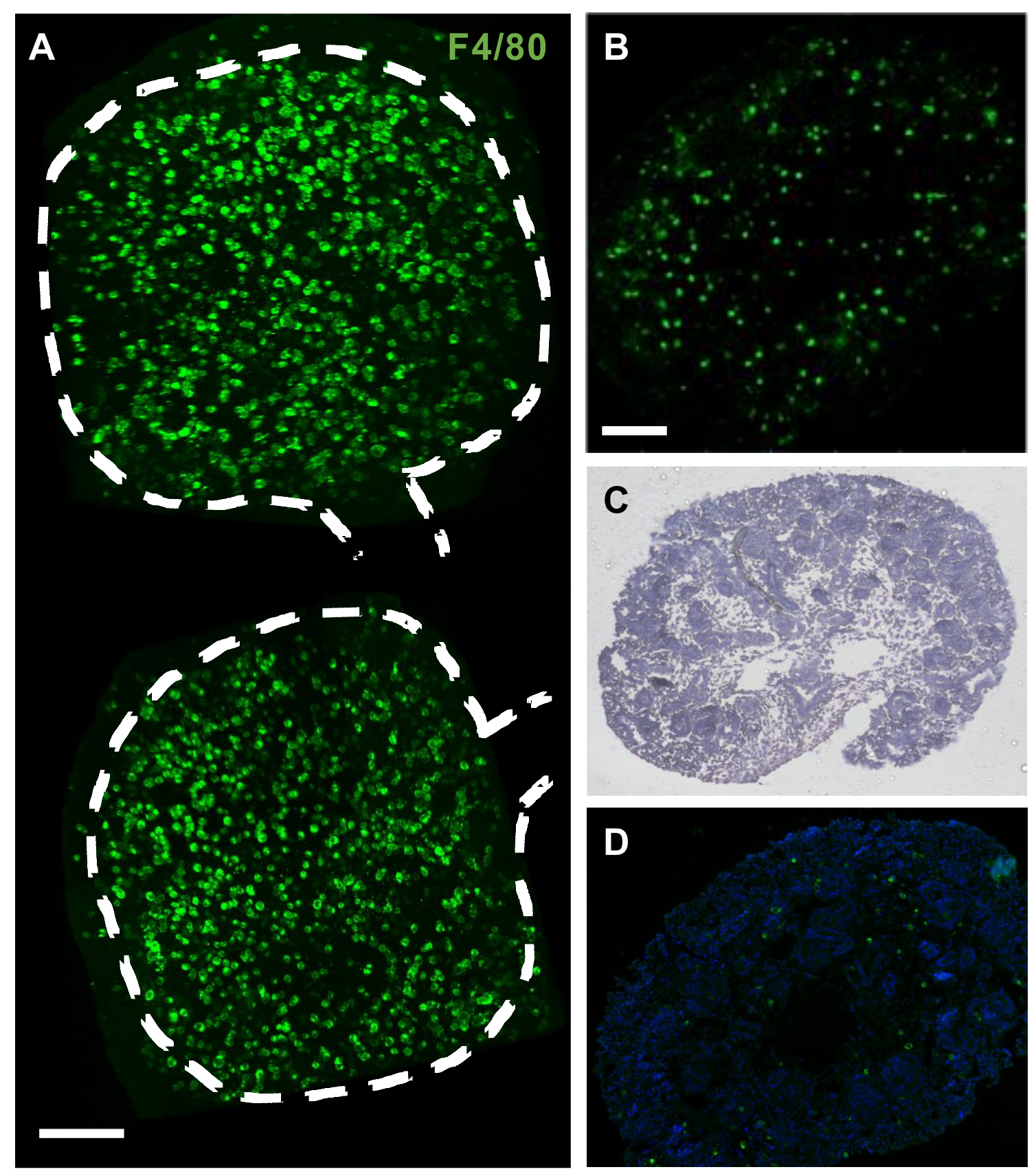

Figure 6. Correlative histology and IHC of BABB-cleared material. A) 3D-

reconstructions, performed in IMARIS (version 8.2, Bitplane) of an embryonic day 14.5 kidney stained with F4/80 (1:50; BioRad; MCAP497). The image was taken using a 25x multi-immersion objective. Two rotated views are provided, with white dashes delineating the metanephros and ureter for orientation B) An optical slice of the same kidney, using ImageJ / FIJI. C) The same kidney, post-paraffin embedding, sectioning (at $5 \mu \mathrm{m}$ ) and staining with Periodic acid-Schiff reagent. D) An alternative section of the kidney, processed with Hoescht and visualized under a fluorescence microscope. Scale bars, $100 \mu \mathrm{m}$. 